\title{
Validación y fiabilidad del cuestionario de desarrollo emocional en Adultos (CDE-A35)
}

\section{Validation and reliability of the Emotional Development Questionnaire in Adults (CDE-A35)}

\author{
Núria PÉREZ-EsCOdA ${ }^{2}$ \\ Universidad de Barcelona, España \\ nperezescoda@ub.edu \\ https://orcid.org/0000-0001-6314-2792 \\ Albert Alegre Rosselló \\ East Stroudsburg University, USA \\ merchalbert@yahoo.es \\ https://orcid.org/0000-0002-7436-1728 \\ ÈLIA LÓPEZ-CASSÀ \\ Universidad de Barcelona, España \\ elialopez@ub.edu \\ https://orcid.org/0000-0003-3870-8533
}

\section{Resumen:}

En este artículo se presenta el estudio de validación de un instrumento para la medición de la inteligencia emocional rasgo. Se trata del CDE-A35, cuestionario de desarrollo emocional de adultos elaborado a partir del modelo pentagonal de la competencia emocional. Se han realizado diversos estudios para demostrar la fiabilidad y la validez del instrumento. Entre ellos se ha realizado el cálculo del coeficiente de

\begin{abstract}
:
This article presents the validation of an instrument for the measurement of trait emotional intelligence. More specifically, the instrument validated is the CDE-A35, an adult emotional development questionnaire elaborated from the pentagonal model of emotional competence. Various studies have been carried out to demonstrate the reliability and validity of the instrument. These include the calculation of
\end{abstract}

1 Como referenciar este artículo (How to reference this article):

Pérez-Escoda, N., Alegre Rosselló, A., \& López-Cassà, E. (2021). Validación y fiabilidad del cuestionario de desarrollo emocional en Adultos (CDE-A35). Educatio Siglo XXI, 39(3), 37-60. https://doi.org/10.6018/educatio.422081

2 Dirección para correspondencia (Correspondence address):

Núria Pérez-Escoda. Departamento de Métodos de Investigación y Diagnóstico en Educación. Facultad de Educación. Pg. de la Vall d’Hebron, 171. 08035, Barcelona (España) 
consistencia interna, estudios factoriales (AFC y AFE), estudios correlacionales con otras medidas de inteligencia emocional, habilidades sociales y personalidad, y se ha efectuado un estudio de regresión. El estudio sugirió la supresión y reubicación de algunos elementos quedando el instrumento definitivo constituido por 35 ítems organizados en cinco factores: conciencia emocional, regulación, competencias sociales, autonomía emocional y competencias de vida y bienestar, coincidentes con las dimensiones de la competencia emocional en el modelo teórico. Las destacables correlaciones con otras pruebas de inteligencia emocional (TElque y CTI), así como con las dimensiones de la prueba de personalidad (NEO-FFI) y con el Inventario de habilidades sociales (EHS), han confirmado su validez convergente. Asimismo, mediante los análisis de regresión se ha confirmado la validez incremental del CDE-A35 sobre otras pruebas ya establecidas. El CDE-A35 es un cuestionario bien fundamentado teóricamente y con buenas características psicométricas, por lo que se considera óptimo para evaluar la inteligencia emocional rasgo en adultos.

\section{Palabras clave:}

Cuestionario; emoción; inteligencia emocional; validación de cuestionarios. the questionnaire's internal consistency coefficient, factor studies (AFC and AFE), correlation studies with other measures of emotional intelligence, social skills and personality, and a regression study. The present validation suggested the suppression and relocation of some elements, leading to a definitive instrument consisting of 35 items organized within five factors: emotional awareness, regulation, social skills, emotional autonomy and life and well-being skills. These five factors coincide with the dimensions of emotional competence in the theoretical model. The remarkable correlations with other emotional intelligence tests (TElque and $\mathrm{CTI}$ ), as well as with the dimensions of the personality test NEO-FFI and with the Inventory of Social Skills (EHS), confirmed its convergent validity. Furthermore, the regression analysis performed confirmed the incremental validity of the CDE-A35 over other tests already established. The CDE-A35 is a sound theoretically-based questionnaire with rigorous psychometric characteristics, and is therefore considered optimal for evaluating trait emotional intelligence in adults.

\section{Keywords:}

Questionnaires; emotional development; emotion; reliability; validity.

\section{Résumé:}

Cet article présente l'étude de validation d'un instrument de mesure de I'Intelligence émotionnelle des traits. II s'agit du CDE-A35, Questionnaire sur le développement émotionnel des adultes élaboré sur la base du modèle pentagonal de la compétence émotionnelle. Diverses études ont été menées pour démontrer la fiabilité et la validité de I'instrument. Parmi eux le calcul du coefficient de cohérence interne, des études factorielles (AFC et AFE), des études des corrélations avec d'autres mesures de l'intelligence émotionnelle, des compétences sociales et de la personnalité, et une étude de régression ont été réalisées. L'étude a suggéré la suppression et la relocalisation de certains éléments, laissant l'instrument définitif composé de 35 éléments organisés en cinq facteurs: conscience émotionnelle, régulation, compétences sociales, autonomie émotionnelle et compétences de vie et de bien-être, coïncidant avec les dimensions de la compétence émotionnelle dans le modèle théorique. Les corrélations remarquables avec d'autres tests d'intelligence émotionnelle (TElque et $\mathrm{CTI}$ ), ainsi qu'avec les dimensions du test de personnalité (NEO-FFI) et avec I'Inventaire des compétences sociales (EHS), ont confirmé leur validité convergente. De plus, l'analyse de régression a confirmé la validité incrémentielle du CDE-A35 par rapport à d'autres tests déjà établis. Le CDE-A35 est un questionnaire théoriquement bien fondé avec de bonnes caractéristiques psychométriques, c'est pourquoi il est considéré comme optimal pour évaluer l'intelligence émotionnelle 
des traits chez l'adulte.

\section{Mots-clés:}

Questionnaire; émotion; intelligence émotionnell; validation des questionnaires.

Fecha de recepción: 07-04-2020

Fecha de aceptación: 03-06-2020

\section{Introducción}

La explosión de interés e investigación en inteligencia emocional (IE) acontecida en las últimas décadas provocó una cierta confusión sobre la definición exacta de la inteligencia emocional y sobre la forma de medirla. Actualmente se proponen dos tipos de inteligencia emocional (O'Connor, Hill, Kaya y Martin, 2019; Petrides, Sanchez-Ruiz, Siegling, Saklofske y Mavroveli, 2018; Siegling, Saklofske y Petrides, 2015). La primera, denominada inteligencia emocional de capacidad (IEC) trata de las habilidades cognitivas relacionadas con la emoción y se mide con tests que evalúan el desempeño óptimo de la persona. La segunda inteligencia, la inteligencia emocional de rasgo (IER) se refiere a la tendencia de la persona a responder y comportarse de forma efectiva en situaciones con contenido emocional. Esas tendencias se miden con cuestionarios de autoinforme.

Se considera que la IER constituye una dimensión transversal de la personalidad (Alegre; Pérez-Escoda y López-Cassà, 2019, Furnham y Pérez-González, 2016, Pérez-González y Sánchez-Ruiz, 2014 y Petrides, Mikolajczak, Mavroveli, Sanchez-Ruiz,). Para medir la IEC existen unos pocos instrumentos sólidamente fundamentados en un marco teórico bien estructurado y justificado. Sin embargo, para la medición de la IER, durante años, los instrumentos disponibles han recibido críticas centradas primordialmente en la falta de un marco teórico claro y de fundamentos empíricos firmes (Bisquerra, Pérez-González y García Navarro 2015; Sánchez-Teruel y Robles-Bello, 2018).

Como respuesta a esta crítica se ha desarrollado el Cuestionario de Desarrollo Emocional de Adultos (CDE-A35) a partir del modelo pentagonal de competencias emocionales de Bisquerra y Pérez (2007), el cual posee un dilatado uso en educación emocional, ya que a partir de él se han desarrollado numerosos programas e investigaciones en la 
última década. Este modelo, conjuga, desde un enfoque integrador, las aportaciones derivadas de los estudios de la IE pero también de otros fundamentos teóricos como los de las inteligencias múltiples, las investigaciones sobre el bienestar y el fluir, estudios sobre la autoestima y las habilidades sociales, la psicología positiva, neurociencia, etc. (Bisquerra y Mateo, 2019; Bisquerra, Pérez-González y García Navarro 2015), que no contemplan otros modelos de la IE.

A partir de ello, se concibe la competencia emocional como el conjunto de conocimientos, capacidades, habilidades y actitudes necesarias para comprender, expresar y regular de forma apropiada los fenómenos emocionales (Bisquerra y Pérez, 2007). Este enfoque integra el saber, el saber hacer y el saber ser.

Tabla 1

Modelo Pentagonal de Competencia Emocional del GROP

\begin{tabular}{ll}
\hline Dimensión & Descripción \\
\hline Conciencia emocional & $\begin{array}{l}\text { Capacidad para tomar conciencia de las propias } \\
\text { emociones, incluyendo la habilidad para captar el } \\
\text { clima emocional de un contexto determinado. }\end{array}$ \\
\hline
\end{tabular}

Regulación emocional Capacidad para utilizar las emociones de forma adecuada. Supone el tomar conciencia de la relación entre emoción, cognición y comportamiento; tener buenas estrategias de "afrontamiento", capacidad para autogenerarse emociones

Autonomía emocional La autonomía emocional incluye un conjunto de características relacionadas con la autogestión emocional, entre las que se encuentran la autoestima, actitud positiva en la vida, responsabilidad, capacidad para analizar críticamente las normas sociales, capacidad para buscar ayuda y recursos, así como la autoeficacia personal.

Competencias sociales Las competencias sociales se refieren a la capacidad para mantener buenas relaciones con otras personas. Esto implica dominar las habilidades sociales básicas, comunicación efectiva, respeto, actitudes prosociales, y asertividad.

Competencias para la Capacidad para adoptar comportamientos aprovida y el bienestar piados y responsables para la solución de problemas personales, familiares, profesionales y sociales, orientados hacia la mejora del bienestar de vida personal y social.

Adaptado de Pérez-Escoda y Filella (2019). 
El modelo teórico, contempla cinco dimensiones de la competencia emocional: conciencia emocional, regulación emocional, competencia social, autonomía emocional y competencias para la vida y el bienestar. Cada una de ellas se divide a su vez en microcompetencias. En la tabla 1 se presenta un resumen del modelo de educación emocional del Grupo de Investigación en orientación Psicopedagógica (GROP). Los expertos coinciden al aceptar la importancia del desarrollo de las competencias emocionales mediante intervenciones educativas (Bisquerra y Mateo, 2019; Durlak, Domitrovich, Weissberg y Gullotta, 2015; Keefer, Parker y Saklofske, 2018; Osher, Kidron, Brackett, Dymnicki, Jones y Weissberg, 2016).

En este sentido, desde el modelo de competencia emocional presentado se cuenta con numerosas aplicaciones educativas que avalan su solidez y utilidad (Pérez-Escoda y Filella, 2019).

El CDE-A35 es un cuestionario de autoinforme basado en el marco teórico presentado. Consta de 35 ítems con una estructura jerárquica de cinco factores de orden inferior y un factor de orden superior. El factor de orden superior es la IE de la persona mientras que los factores de orden inferior se corresponden con las cinco dimensiones del modelo pentagonal. Utiliza una escala de Likert de once opciones de respuesta de cero a diez. El CDE-A35 responde a la necesidad de ofrecer un instrumento riguroso, adaptado a la población de habla hispana, que permita evaluar el nivel de IER. En la tabla 2 se analizan sus características en relación con otras pruebas de reconocido prestigio.

Tabla 2

Comparación entre instrumentos de medida de la IE

\begin{tabular}{cccc}
\hline & IEC: MSCEIT & IER: TEiQue & IER: CDE-A35 \\
\hline Tipo de prueba & $\begin{array}{c}\text { Preguntas de } \\
\text { desempeño óptimo }\end{array}$ & Autoinforme & Autoinforme \\
\hline $\begin{array}{ccc}\text { Fundamentación conceptual } \\
\text { teórica }\end{array}$ & Fuerte & Débil & Fuerte \\
$\begin{array}{c}\text { Propiedades Psicométricas } \\
\text { Relación con la personalidad }\end{array}$ & Débiles & Buenas & Buenas \\
Rendimiento medido & Débil & Fuerte & Fuerte \\
Posibilidad de engaño & No & Habitual & Habitual \\
\hline
\end{tabular}

Adaptación de Kotsou (2019, p. 25). 
Dado el interés de un cuestionario de estas características, es importante aportar datos que confirmen su validez y fiabilidad para medir la inteligencia emocional de los adultos. Esto incluye mostrar evidencia de la validez convergente, de criterio, e incremental del cuestionario en una muestra amplia y diversa.

En consecuencia, el propósito del estudio actual es testar la fiabilidad del CDE-A35 y su validez en una muestra heterogénea de adultos. En concreto, se proponen seis objetivos:

(1) Evaluar la fiabilidad de las dimensiones o factores del CDE-A35.

(2) Examinar la estructura propuesta de cinco factores de acuerdo con el modelo pentagonal.

(3) Evaluar la validez convergente y divergente mediante la relación entre el CDE-A35 y otras medidas de IE y una medida de habilidades sociales.

(4) Confirmar la relación de la IER con las cinco grandes dimensiones de la personalidad.

(5) Testar la validez predictiva del CDE-A35 en relación con la satisfacción con la vida.

(6) Explorar la validez incremental del CDE-A35 sobre las cinco dimensiones de la personalidad

\section{Método}

\section{Participantes}

Para validar el CDE-A35 se ha realizado un estudio con 3.073 personas a las que tuvieron acceso los investigadores y que fueron invitadas a participar libremente. Un $48.7 \%$ formado por estudiantes de grados y másteres en facultades de psicología y educación de diversas universidades españolas y el $51.3 \%$ restante adultos voluntarios. En su mayoría (76\%), eran mujeres. La edad de los participantes osciló entre los 18 y 67 años y 35.28 de media (DT=11,27). El 70.7\% contaba con estudios universitarios y un $82.4 \%$ trabajaban durante el estudio. 
Tabla 3

Características sociodemográficas de la muestra total

\begin{tabular}{lcccccc}
\hline & Muestra & Estudiantes & $\begin{array}{c}\text { Otros } \\
\text { adultos }\end{array}$ & Edad Media & $\begin{array}{c}\text { Estudiantes } \\
\text { universitarios }\end{array}$ & Empleados \\
\hline Hombres & $\begin{array}{c}731 \\
(23.8 \%)\end{array}$ & 356 & 371 & $\begin{array}{c}38.3 \\
(\mathrm{DT}=11.1)\end{array}$ & 522 & 1.928 \\
\hline Mujeres & $\begin{array}{c}2.342 \\
(76.2 \%)\end{array}$ & 1.141 & 1205 & $\begin{array}{c}33.9 \\
(\mathrm{DT}=11.0)\end{array}$ & 1.651 & 604 \\
\hline Total & 3.073 & $\begin{array}{c}1.497 \\
(48.7 \%)\end{array}$ & $\begin{array}{c}1.576 \\
(51.3 \%)\end{array}$ & $\begin{array}{c}35.3 \\
(\mathrm{DT}=11.3)\end{array}$ & $\begin{array}{c}2.173 \\
(70.7 \%)\end{array}$ & $\begin{array}{c}2.532 \\
(82.4 \%)\end{array}$ \\
\hline
\end{tabular}

Fuente: elaboración propia.

Los participantes respondieron una batería de cuestionarios y recibieron un informe con sus resultados. Por limitaciones de tiempo y disponibilidad de los instrumentos, todos los participantes se aplicaron el CDE-A35, aunque no todos respondieron al resto de cuestionarios. Por ello, para determinados análisis se han utilizado muestras parciales.

En cuanto al tercer objetivo (validez convergente y divergente) se tomaron diversas muestras parciales: a) 191 personas que respondieron el TeiQue, de las cuales el 79\% fueron mujeres, mayoritariamente con estudios universitarios y trabajo. La edad osciló entre los 20 y 55 años con 29.de media (DT=7.89); b) 72 participantes respondieron el CTI. El 90\% fueron mujeres, con edad entre 19 y 36 años, con una media de 21 años (DT=2.74). El 76\% tenían formación de bachillerato y el resto universitaria. El 56.9\% trabajaba durante el estudio; c) 580 personas respondieron el EHS, el $86 \%$ de las cuales eran mujeres con edades entre los 18 y 64 años, con una media de 22.13 años (DT=4.71). El 75.5\% tenía formación de bachillerato y el resto universitaria y el $62.8 \%$ trabajaba.

Para el estudio del cuarto objetivo (relación con rasgos de personalidad) y del sexto objetivo (validez incremental), se contó con una muestra parcial de 541 participantes, el $86.9 \%$ de sexo femenino y edades entre los 19 y los 64 años, con una media de 30.04 años (DT=9.53). El 70,2\% tenían formación universitaria y el $71.9 \%$ tenía trabajo. Finalmente, para el quinto objetivo (predicción de la satisfacción con la vida), la muestra parcial fue de 2.032 participantes, mayoritariamente mujeres (81.1\%), de edad media 34.78 oscilando entre los 18 y 67 años (DT=11.19). El $74.8 \%$ con formación universitaria y el $82.5 \%$ con trabajo. 


\section{Procedimiento}

Se utilizó el software AMOS 20 para realizar los diferentes análisis estadísticos. La estructura teórica del CDE-A35 de cinco dimensiones se testó a través de un análisis factorial confirmatorio (AFC). El test de adecuación de la muestra de Kaiser-Meyer-Olkin fue de 0.932, y el test de esfericidad de Bartlett resultó significativo ( $p<0.001)$, permitiendo la realización de este análisis.

Para confirmar el ajuste del modelo propuesto a los datos recogidos utilizamos dos índices de ajuste relativo -el CFI y el IFI- un índice de ajuste de parsimonia -el PCFI- y un índice de no centralidad -el RMSA-. Se considera un ajuste aceptable del modelo cuando el CFI e IFI >.90 (Kline, 1998) o un ajuste óptimo cuando >.95 (Hu y Bentler, 1999), también un buen ajuste cuando RMSEA $<.80$ (Cheing, Vong, Chan, Ditchman, Brooks y Chan, 2014) y el PCFI $>.50$ (Khayyam et al., 2014).

Diversos autores (Floyd y Widaman, 1995; Quintana y Maxwell, 1999) han puesto de manifiesto que un análisis factorial confirmatorio el cual utiliza todos los ítems, es excesivamente riguroso porque los ítems individuales tienen poca fiabilidad, bajas intercorrelaciones y correlaciones limitadas con otras variables, y como consecuencia se hace muy difícil confirmar el ajuste del modelo a los datos. La alternativa es la creación de parcelas de ítems.

Esta técnica consiste en utilizar la media aritmética de dos o más ítems en sustitución de éstos. Ello permite aumentar la fiabilidad, mejorar las comunalidades, aumentar el grado de varianza factorial y lograr distribuciones más cercanas a la normal (Little, Rhemtulla, Gibson y Schoemann, 2013; McCallum, Widaman, Zhang y Hong, 1999).

Desde esta perspectiva metodológica-analítica, en este estudio se crearon parcelas de ítems para realizar un nuevo AFC y así garantizar un test superior de los parámetros del modelo estructural, de forma que los constructos estuvieran definidos precisamente, seguimos la recomendación de Little (2013) en cuanto a componer tres parcelas de ítems para cada variable.

Los ítems fueron asignados aleatoriamente a las parcelas. Para cada parcela se calculó la media de los ítems que lo componían. La matriz de covarianza entre las parcelas se utilizó como input en el análisis factorial, y se realizó una estimación de máximas probabilidades (Hu y Bentler, 1995). 
Adicionalmente, se calculó la fiabilidad de cada uno de los instrumentos utilizados en este estudio, incluyendo las subescalas de cada uno, utilizando el coeficiente $\alpha$ de Cronbach. Después se aplicó el coeficiente de correlación de Pearson para comprobar la validez convergente y el análisis de regresión para comprobar la validez de criterio, e incremental (sobre las dimensiones de la personalidad) del cuestionario CDE-A35.

\section{Instrumentos}

Competencia emocional. Se utilizó el CDE-A35. Este cuestionario ofrece una medida de la competencia emocional que puede subdividirse en cinco escalas: la primera denominada conciencia emocional, contiene ocho ítems referentes al conocimiento de las propias emociones y las emociones de los demás (p.ej., Sé poner nombre a las emociones que siento). La segunda denominada regulación emocional, agrupa ocho ítems relacionados con la gestión emocional (p.ej., A menudo me dejo llevar por la rabia y actúo bruscamente).

La tercera escala denominada autonomía emocional, agrupa seis ítems referentes a la autoconfianza, autonomía, e independencia. (p.ej., Me siento herido fácilmente cuando los otros critican mi conducta o trabajo). La competencia social es la cuarta, tiene cinco ítems relativos a la capacidad de lidiar con relaciones interpersonales (p.ej., Hablar con otras personas poco conocidas me resulta difícil, hablo poco y siento nerviosismo).

La quinta escala denominada competencias de vida y bienestar tiene ocho ítems relativos al del bienestar de la persona y la autosatisfacción (p.ej., Estoy satisfecho con mi manera de afrontar la vida y ser feliz).

Inteligencia emocional rasgo (IER). Se utilizó el Trait Emotional Intelligence Questionnaire (TEIQue) desarrollado por Petrides, (2009), y considerado como una de las más comprensivas, válidas y fiables evaluaciones de la inteligencia emocional global como conjunto de disposiciones de personalidad que favorecen un comportamiento emocionalmente inteligente (Andrei, Siegling, Aloe, Baldaro y Petrides, 2016; FernándezAbascal, y Martín-Díaz, 2015; Pérez-González y Sánchez-Ruiz, 2014). Consta de 153 elementos, clasificados en una escala Likert de 7 puntos, y cubre 15 facetas distintas agrupadas en cuatro factores: bienestar, emocionalidad, sociabilidad y autocontrol. Sánchez-Ruiz, Pérez-González y Petrides, (2010) informan que la consistencia interna de las escalas es 
$\alpha=.84, \alpha=.73, \alpha=.70$ y $\alpha=.75$, respectivamente, siendo $\alpha=.90$ para la IE global. En nuestro estudio la consistencia interna para la escala total fue de: $\alpha=.94$.

Personalidad. Se utilizó el NEO PI-R de Costa y McCrae (1989) en su versión abreviada NEO-FFI de la adaptación española de Cordero, Pamos y Seisdedos (2008). Se trata de uno de los instrumentos más reconocidos para la evaluación de la personalidad. Se basa en la estructura de los Ilamados "cinco grandes" de la personalidad: neuroticismo, apertura, extraversión, responsabilidad y amabilidad. El NEO-FFI consta de 60 elementos y se responde en una escala Likert de cinco puntos. La fiabilidad y validez del instrumento han sido demostradas en numerosos estudios para diversas culturas, obteniendo una consistencia interna entre 0.72 y 0.92 (vease, por ejemplo, Magalhães et al., 2014). En este estudio las fiabilidades de las cinco dimensiones fueron .89, .82, .78, .69, y .81 respectivamente.

Satisfacción con la vida. Se mide con la escala SWLS de Diener, Emmons, Larsen y Griffin (1985). Se trata de la medida de satisfacción vital más utilizada en la literatura científica. Consiste en 5 afirmaciones respecto a las que se indica el nivel de acuerdo o desacuerdo en una escala tipo Likert de 7 puntos. Presenta buenas propiedades psicométricas con una alta consistencia interna que oscila entre .79 y .89 según los estudios (Diener, Emmons, Larsen y Griffin, 1985; Diener y González, 2011), y se dispone también de suficiente evidencia empírica acerca de la validez de la escala en muestras españolas (Vázquez, Duque, de Hervas, 2013). En el presente estudio la fiabilidad de la escala fue de $=.84$.

Pensamiento Constructivo, relacionado con el ajuste emocional y con el éxito en la vida. Se utilizó el Inventario CTI elaborado por Epstein en 1987, en la versión castellana adaptada por Cordero (2001). Es un instrumento para adolescentes y adultos que consta de 108 ítems con 5 opciones de respuesta. Evalúa el pensamiento constructivo y destructivo que se produce de manera automática en la vida diaria. Contiene una dimensión general (PGC) y seis escalas (eficacia, emotividad, pensamiento supersticioso, pensamiento esotérico, rigidez e ilusión) y varias subescalas que integran el tercer nivel de generalidad. También incluye otras dos escalas de validación (deseabilidad y validez). La fiabilidad de las escalas del nivel 1 y 2 utilizadas en este estudio fueron superiores a $\alpha>.70$.

Aserción y habilidades sociales. Se aplicó la Escala EHS (Gismero, 2000). Este cuestionario examina la conducta habitual de adolescentes 
y adultos en situaciones específicas y valora hasta qué punto las habilidades sociales inciden en sus actitudes. El EHS está compuesto por 33 elementos con los que mide 6 factores: autoexpresión de opiniones y sentimientos en situaciones sociales; defensa de los propios derechos como consumidor; expresión de enfado o disconformidad; decir no y cortar interacciones; hacer peticiones y iniciar interacciones positivas con el sexo opuesto. Proporciona una puntuación global sobre habilidades sociales. Su fiabilidad global en el presente estudio fue $\alpha=.85$ y en los factores fueron $.74, .56, .55, .70,45, .62$ respectivamente.

Datos demográficos. Se utilizó un breve cuestionario incluyendo algunas preguntas sobre la edad (número de años), el género (hombre o mujer), el nivel de estudios (estudios básicos, ESO, bachillerato, ciclo formativo de grado medio, de grado superior, estudios universitarios de primer ciclo, de segundo ciclo, doctorado o formación universitaria de tercer ciclo) y situación laboral (no trabajo, sí trabajo).

\section{Resultados}

\section{Estructura factorial del CDE-A35}

Como se ha comentado, el CDE-A35 se fundamenta en un modelo de IE compuesto de cinco dimensiones: conciencia emocional, regulación emocional, competencia social, autonomía emocional y competencias de vida y bienestar. El análisis factorial confirmatorio con parcelación aleatoria de ítems fue positivo. El ajuste del modelo alcanzó los valores de aceptación requeridos: $\chi^{2}(74, \mathrm{~N}=3073)=531.40, \mathrm{CFI}=.95, \mathrm{IFI}=.95$, RMSEA $=.06, \mathrm{PCFI}=.67$.

Las cargas factoriales de las diferentes dimensiones fueron respectivamente: conciencia emocional $(\beta=.57)$, regulación emocional $(\beta=.67)$, autonomía emocional $(\beta=.79)$, competencia social $(\beta=.69)$ y competencias de vida $(\beta=.88)$. Adicionalmente, se realizó un análisis factorial exploratorio para comprobar el peso de cada ítem en su escala original (ver tabla 4). La varianza explicada por los cinco factores es del $42.70 \%$.

Las cinco escalas mostraron fuertes correlaciones: con el coeficiente global de IE .65, para la conciencia emocional .74 para regulación emocional, .74 para la autonomía emocional, .66 para competencia social y .80 para competencias de vida. 
Tabla 4

Pesos factoriales de los ítems incluidos en cada factor

\begin{tabular}{|c|c|c|c|c|c|}
\hline & \multicolumn{5}{|c|}{ Componentes } \\
\hline & 1 & 2 & 3 & 4 & 5 \\
\hline Ítem 7 & .726 & & & & \\
\hline Ítem 44 & .665 & & & & \\
\hline Ítem 5 & .658 & & & & \\
\hline Ítem 13 & .647 & & & & \\
\hline Ítem 11 & .531 & & & & \\
\hline Ítem 45 & .506 & & & & \\
\hline Ítem 8 & .491 & & & & \\
\hline Ítem 36 & .394 & & & & \\
\hline Ítem 31 & & .651 & & & \\
\hline Ítem 30 & & .602 & & & \\
\hline Ítem 47 & & .595 & & & \\
\hline Ítem 23 & & .578 & & & \\
\hline Ítem 46 & & .500 & & & \\
\hline Ítem 43 & & .467 & & & \\
\hline Ítem 14 & & .460 & & & \\
\hline Ítem 22 & & .433 & & & \\
\hline Ítem 26 & & & .549 & & \\
\hline Ítem 37 & & & .474 & & \\
\hline Ítem 38 & & & .383 & & \\
\hline Ítem 16 & & & .275 & & \\
\hline Ítem 32 & & & .242 & & \\
\hline Ítem 18 & & & & .626 & \\
\hline Ítem 4 & & & & .585 & \\
\hline Ítem 48 & & & & .560 & \\
\hline Ítem 40 & & & & .551 & \\
\hline Ítem 2 & & & & .524 & \\
\hline Ítem 12 & & & & .510 & \\
\hline Ítem 34 & & & & & .654 \\
\hline Ítem 29 & & & & & .633 \\
\hline Ítem 15 & & & & & .625 \\
\hline Ítem 21 & & & & & .579 \\
\hline Ítem 3 & & & & & .562 \\
\hline Ítem 24 & & & & & .501 \\
\hline Ítem 33 & & & & & .394 \\
\hline Ítem 1 & & & & & .356 \\
\hline
\end{tabular}

Nota. $\mathrm{N}=1510$. Fuente: Elaboración propia. 


\section{Fiabilidad Interna del CDE-A35}

Todas las dimensiones de la competencia emocional medidas por el CDE-A35 mostraron fiabilidades internas adecuadas. Concretamente, las fiabilidades medidas con el $\alpha$ de Cronbach fueron: conciencia emocional $\mathrm{CE}=.78$, regulación emocional $\mathrm{RE}=.77$, autonomía emocional $\mathrm{AU}=.77$, competencia social $\mathrm{CS}=.64$ y competencias de vida y bienestar $\mathrm{CV}=.80$, y la escala completa $\mathrm{CET}=.89$ (ver tabla 5 ).

Tabla 5

Datos Descriptivos CDE-A35

\begin{tabular}{lccccc}
\hline \multicolumn{1}{c}{ Escalas } & Mínimo & Máximo & Media & DT & $\alpha$ \\
\hline Conciencia emocional & 14 & 80 & 58.61 & 9.85 & .78 \\
Regulación emocional & 8 & 80 & 50.09 & 11.40 & .77 \\
Autonomía emocional & 0 & 60 & 28.08 & 9.99 & .77 \\
Competencia social & 0 & 50 & 27.63 & 8.48 & .64 \\
Competencias de Vida & 3 & 80 & 59.11 & 11.26 & .80 \\
Competencia Emocional Total & $\mathbf{7 2}$ & $\mathbf{3 3 0}$ & $\mathbf{2 2 3 . 5 4}$ & $\mathbf{3 6 . 7 3}$ & $\mathbf{. 8 9}$ \\
\hline
\end{tabular}

Nota. $\mathrm{N}=3073$. Fuente: Elaboración propia.

\section{Validez Convergente}

Para investigar la validez convergente del cuestionario se analizó la relación entre el CDE-A35 y otras medidas de IE y habilidad social tales como el TEIQue, el CTI y el EHS. EI TEIQue consta de cuatro factores bienestar, autocontrol, emocionalidad y sociabilidad. Las correlaciones pueden verse en la tabla 5. Observamos una muy elevada correlación entre las medidas globales de ambas escalas CDEA35 i TElque ( $r=.86 ; p<.001)$.

Asimismo, todas las dimensiones del CDE-A35 correlacionan de forma elevada y positiva con la IER medida con el TElque. Adicionalmente, la escala conciencia emocional destaca por su fuerte correlación con el factor emocionalidad $(r=.82 ; p<.001)$, la escala regulación emocional con el factor autocontrol $(r=.66 ; p<.001)$, la escala competencia social con el factor sociabilidad $(r=.57 ; p<.001)$, la escala autonomía emocional con los factores autocontrol $(r=.50 ; p<.001)$ y bienestar $(r=.43$; $\mathrm{p}<.001$ ) y la escala competencias de vida y bienestar con el factor bienestar $(r=.79 ; p<.001)$. Todas las escalas del CDE-A35 mantienen correlaciones positivas con todas las escalas del TElque con la única excepción de la escala regulación y el factor sociabilidad. 
En cuanto a la relación con el EHS, todas las escalas del CDE-A35 correlacionaron con el coeficiente global de habilidad social y con cada uno de sus componentes (ver tabla 6). La correlación entre las puntuaciones totales de ambas escalas fue destacable $(r=.57 ; p<.001)$ y la dimensión competencia social medida con el CDE-A35 arrojó la mayor correlación con el coeficiente global de habilidad social $(r=.57 ; p<.001)$.

Para comprobar la validez convergente del CDE-A35 también se calculó la correlación entre la IE medida con este instrumento y la IE medida con el $\mathrm{CTI}(\mathrm{r}=.81 ; \mathrm{p}<.000)$ siendo muy alta y significativa. Además, también destaca una relación fuerte y positiva de todas las dimensiones del CDE-A35 y del total con la escala de afrontamiento emotivo $(r=.81 ; p<.000)$ y que incluye aspectos como la autoaceptación, la ausencia de sobregeneralización negativa, la ausencia de hipersensibilidad, y la ausencia de rumiaciones.

La conciencia emocional además correlaciona positivamente con el afrontamiento conductual $(\mathrm{r}=.45 ; \mathrm{p}<.001)$ que incluye aspectos tales como el pensamiento positivo, la orientación a la acción, y la responsabilidad. Por el contrario, todas las escalas de la IE medidas con el CDE-A35 correlacionan negativamente con el pensamiento mágico, y el categórico, aunque las correlaciones de la conciencia emocional no fueron significativas. La competencia de vida y bienestar también correlacionó negativamente con el pensamiento esotérico $(r=-.32 ; \mathrm{p}<.05)$. Ninguna dimensión del CDE-A35 correlacionó significativamente con el optimismo ingenuo (ver tabla 5).

Tabla 6

Correlaciones con las variables del TElque y EHS

\begin{tabular}{lcccccc}
\hline \multicolumn{7}{c}{ CDE-A35 } \\
& Total & enciencia & Regulación & Autonomía & \multicolumn{2}{c}{ Competen- C.de vida } \\
& emocional & emocional & cia Social & bienestar \\
\hline TElque_b & $.737^{* *}$ & $.310^{* *}$ & $.584^{* *}$ & $.434^{* *}$ & $.483^{* *}$ & $.789^{* *}$ \\
TElque_e & $.556^{* *}$ & $.815^{* *}$ & $.300^{* *}$ & $.159^{*}$ & $.388^{* *}$ & $.436^{* *}$ \\
TElque_s & $.462^{* *}$ & $.272^{* *}$ & .141 & $.306^{* *}$ & $.568^{* *}$ & $.364^{* *}$ \\
TElque_a & $.612^{* *}$ & $.232^{* *}$ & $.657^{* *}$ & $.495^{* *}$ & $.293^{* *}$ & $.428^{* *}$ \\
EHS & $.574^{* * *}$ & $.352^{* * *}$ & $.192^{* * *}$ & $.540^{* * *}$ & $.570^{* * *}$ & $.422^{* * *}$ \\
Factor 1 & $.578^{* * *}$ & $.372^{* * *}$ & $.177^{* * *}$ & $.491^{* * *}$ & $.628^{* * *}$ & $.436^{* * *}$ \\
Factor 2 & $.295^{* * *}$ & $.133^{* * *}$ & $.095^{*}$ & $.350^{* * *}$ & $.321^{* * *}$ & $.175^{* * *}$ \\
Factor 3 & $.257^{* * *}$ & $.228^{* * *}$ & -.004 & $.284^{* * *}$ & $.304^{* * *}$ & $.184^{* * *}$ \\
Factor 4 & $.421^{* * *}$ & $.200^{* * *}$ & $.240^{* * *}$ & $.441^{* * *}$ & $.299^{* * *}$ & $.308^{* * *}$ \\
Factor 5 & $.263^{* * *}$ & $.143^{* * *}$ & $.090^{*}$ & $.277^{* * *}$ & $.236^{* * *}$ & $.197^{* * *}$ \\
Factor 6 & $.442^{* * *}$ & $317^{* * *}$ & $.175^{* * *}$ & $.301^{* * *}$ & $.459^{* * *}$ & $.350^{* * *}$ \\
\hline
\end{tabular}




\begin{tabular}{lcccccc}
\hline & \multicolumn{2}{c}{ CDE-A35 Conciencia Regulación Autonomía } & \multicolumn{2}{c}{ Competen- C.de vida } \\
& Total & emocional & emocional & emocional & cia Social & y bienestar \\
\hline PCG & $.810^{* * *}$ & $.413^{* *}$ & $.572^{* * *}$ & $.749^{* * *}$ & $.462^{* * *}$ & $.676^{* * *}$ \\
EMO & $.812^{* * *}$ & $.291^{*}$ & $.548^{* * *}$ & $.834^{* * *}$ & $.450^{* * *}$ & $.720^{* * *}$ \\
CON & .271 & $452^{* *}$ & .123 & .206 & .107 & .135 \\
MAG & $-.736^{* * *}$ & -.256 & $-.578^{* * *}$ & $-.647^{* * *}$ & $-.382^{* *}$ & $-.685^{* * *}$ \\
CAT & $-.554^{* * *}$ & -.257 & $-.570^{* * *}$ & -.282 & $-.392^{* *}$ & $-.468^{* * *}$ \\
ESO & -.220 & .002 & -.203 & -.120 & -.033 & $-.319^{*}$ \\
OPT & -.091 & .137 & -.018 & -.075 & -.162 & -.201 \\
\hline
\end{tabular}

Nota. $\mathrm{N}=191$ para datos del TElque, $\mathrm{N}=580$ para datos de escala EHS y $\mathrm{N}=72$ para datos del CTI. ${ }^{*} p<.05 * * p<.01 * * p<.001$. TElque_b=Bienestar, TElque_e=Emocionalidad, TElque_ $\mathrm{s}=$ Sociabilidad, TElque_a=Autocontrol. Factor 1: autoexpresión de opiniones, sentimientos en situaciones sociales; Factor 2: defensa de los propios derechos como consumidor; Factor 3: expresión de enfado o disconformidad; Factor 4: decir no y cortar interacciones; Factor 5: hacer peticiones y Factor 6: iniciar interacciones positivas con el sexo opuesto. PCG=Pensamiento constructivo global; $\mathrm{EMO}=$ Afrontamiento emocional; $\mathrm{CON}=$ Afrontamiento conductual; MAG=Pensamiento mágico; $\mathrm{CAT}=$ Pensamiento categórico; $\mathrm{ESO}=$ Pensamiento esotérico; OPT=Optimismo ingenuo. Fuente: Elaboración propia.

\section{Correlaciones con las dimensiones de la personalidad}

Todas las escalas del CDE-A35 correlacionan negativamente con el neuroticismo y positivamente con la extroversión, la amabilidad, la responsabilidad, y la apertura medidas con el NEO-FFI (ver tabla 7). Destaca que la correlación más fuerte se da con el neuroticismo, para todas las escalas excepto para la conciencia emocional cuyas correlaciones más altas de dan con la apertura, y la competencia social cuya máxima correlación se da con la extroversión.

Tabla 7

Correlaciones con medidas de personalidad

\begin{tabular}{lccccc}
\hline & $\mathrm{N}$ & $\mathrm{E}$ & $\mathrm{O}$ & $\mathrm{A}$ & $\mathrm{R}$ \\
\hline Conciencia Emocional & $-.27^{* *}$ & $.32^{* *}$ & $.38^{* *}$ & $.26^{* *}$ & $.32^{* *}$ \\
Regulación emocional & $-.60^{* *}$ & $.20^{* *}$ & .09 & $.52^{* *}$ & $.42^{* *}$ \\
Autonomía emocional & $-.61^{* *}$ & $.26^{* *}$ & $.20^{* *}$ & $.25^{* *}$ & $.26^{* *}$ \\
Competencia social & $-.40^{* *}$ & $.58^{* *}$ & $.31^{* *}$ & $.20^{* *}$ & $.19^{* *}$ \\
Competencias de Vida & $-.68^{* *}$ & $.41^{* *}$ & $.16^{* *}$ & $.28^{* *}$ & $.36^{* *}$ \\
CDE-A35 Total & $\mathbf{- . 7 1 ^ { * * }}$ &. $\mathbf{4 7 *}$ &. $\mathbf{3 0}$ &. $\mathbf{4 2 *}$ &. $\mathbf{4 3}$ \\
\hline
\end{tabular}

Nota. $\mathrm{N}=541 .{ }^{* *} . \mathrm{p}<.01 . \mathrm{N}=$ Neuroticismo, $\mathrm{E}=$ Extraversión, $\mathrm{O}=$ Apertura, $\mathrm{A}=$ Amabilidad, $\mathrm{R}=$ Responsabilidad. Fuente: Elaboración propia. 


\section{Validez de Criterio}

Para testar la validez de criterio del CDE-A35, se efectuó un análisis de regresión de la satisfacción con la vida medida con el SWLS. En un primer paso, se utilizaron las variables demográficas de edad, género, estudios y trabajo más la variable de la IE en la ecuación de regresión. Los resultados muestran, tal y como estaba previsto, que la IE predice la satisfacción con la vida más allá de las variables demográficas mencionadas $(\beta=.36, p<.000)$. La varianza total explicada fue del $14 \%$. En un segundo paso, la IE fue sustituida por sus escalas. Así encontramos que la autonomía emocional, y las competencias de vida predicen la satisfacción con la vida, pero no así la conciencia emocional, la competencia social o la regulación emocional (ver tabla 8).

\section{Validez Incremental}

Para investigar la validez incremental del CDE-A35 sobre otras medidas ya establecidas, se utilizó un análisis de regresión jerárquico. En un primer paso, se introdujeron las variables demográficas. En un segundo paso, se introdujeron las cinco dimensiones de la personalidad medidas por el NEO-FFI. Los resultados mostraron que el neuroticismo, la extroversión, y la responsabilidad predicen la satisfacción con la vida. En un tercer paso, se observa que la IE medida con el CDE-A35 aporta también poder de predicción adicional. En el cuarto paso, la variable IE fue substituida por sus cinco dimensiones. Se observó que las competencias de vida aportaban valor de predicción por encima de las "cinco grandes" dimensiones de la personalidad. Los bajos valores de los factores de inflación de la varianza que se mantuvieron entre 1.04 y 2.85 indicando que no hay problemas de colinealidad entre las variables (ver tabla 9). 
Pérez-Escoda, N., Alegre Rosselló, A. y López-Cassà, È. (2021). Validación y fiabilidad del cuestionario de desarrollo emocional en Adultos (CDE-A35). Educatio Siglo XXI, 39(3), 37-60.

Tabla 8

Análisis de Regresión. Predicción de la satisfacción en la vida a partir de las dimensiones de la inteligencia emocional

\begin{tabular}{lccccc}
\hline \multirow{2}{*}{ Modelo } & \multicolumn{2}{c}{$\begin{array}{c}\text { Coeficientes no } \\
\text { estandarizados }\end{array}$} & $\begin{array}{c}\text { Coeficientes } \\
\text { estandarizados }\end{array}$ & \multirow{2}{*}{$\mathrm{t}$} & Sig. \\
\cline { 2 - 5 } & $\mathrm{B}$ & Error Std. & Beta & & \\
\hline (Constante) & 14.964 & .808 & & 18.520 & .000 \\
Sexo & -.404 & .277 & -.031 & -1.461 & .144 \\
Edad & -.038 & .011 & -.081 & -3.493 & .000 \\
Estudios & .053 & .079 & .015 & .670 & .503 \\
Trabajo & .661 & .299 & .048 & 2.208 & .027 \\
Inteligencia Emocional & .050 & .003 & .358 & 17.229 & .000 \\
\hline (Constante) & 11.899 & .863 & & 13.787 & .000 \\
Sexo & -.217 & .264 & -.016 & -.821 & .412 \\
Edad & -.015 & .010 & -.032 & -1.445 & .149 \\
Estudios & .003 & .074 & .001 & .047 & .963 \\
Trabajo & .463 & .281 & .034 & 1.647 & .100 \\
Conciencia Emocional & -.002 & .014 & -.004 & -.175 & .861 \\
Regulación emocional & .021 & .011 & .046 & 1.953 & .051 \\
Autonomía emocional & .233 & .011 & .511 & 20.333 & .000 \\
Competencia social & .011 & .011 & .021 & .956 & .339 \\
Competencias de Vida & -.063 & .013 & -.122 & -4.882 & .000 \\
\hline
\end{tabular}

Fuente: Elaboración propia

Las ecuaciones de regresión: SV $=18520-0,081$ e + 0,048 + 0,358IE SV = $13,787+0,511 \mathrm{AU}-0,122 \mathrm{CV}$

$\mathrm{SV}$ = Satisfacción en la vida, e = edad, $\mathrm{t}=$ trabajo, IE = Inteligencia emocional, $\mathrm{AU}=$ Autonomía emocional, $\mathrm{CV}=$ Competencias de vida y bienestar 
Tabla 9

Análisis de Regresión. Predicción de la satisfacción en la vida a partir de las dimensiones de la personalidad y de la inteligencia emocional

\begin{tabular}{|c|c|c|c|c|c|}
\hline \multirow[t]{2}{*}{ Modelo } & \multicolumn{2}{|c|}{$\begin{array}{l}\text { Coeficientes no } \\
\text { estandarizados }\end{array}$} & \multicolumn{3}{|c|}{$\begin{array}{c}\text { Coeficientes } \\
\text { estandarizados }\end{array}$} \\
\hline & $\mathrm{B}$ & Error Std. & Beta & $\mathrm{t}$ & Sig. \\
\hline (Constante) & 25.464 & 1.12 & & 22.91 & .000 \\
\hline sexo & -.204 & .646 & -.014 & -.316 & .752 \\
\hline edad & -.045 & .026 & -.085 & -1.71 & .087 \\
\hline Estudios & .155 & .152 & .049 & 1.02 & .309 \\
\hline Trabajo & 1.055 & .490 & .095 & 2.153 & .032 \\
\hline (Constante) & 24.446 & 2.36 & & 10.37 & .000 \\
\hline sexo & -.994 & .564 & -.068 & -1.76 & .079 \\
\hline edad & -.048 & .023 & -.093 & -2.14 & .033 \\
\hline Estudios & .022 & .128 & .007 & .169 & .866 \\
\hline Trabajo & .246 & .415 & .022 & .592 & .554 \\
\hline Neuroticismo & -.219 & .026 & -.388 & -8.46 & .000 \\
\hline Extraversión & .117 & .029 & .166 & 3.99 & .000 \\
\hline Apertura & -.004 & .027 & -.006 & -.165 & .869 \\
\hline Amabilidad & .014 & .036 & .016 & .389 & .697 \\
\hline Responsabilidad & .119 & .030 & .163 & 4.01 & .000 \\
\hline (Constante) & 20.169 & 2.551 & & 7.907 & .000 \\
\hline sexo & -1.058 & .556 & -.072 & -1.901 & .058 \\
\hline edad & -.056 & .022 & -.108 & -2.518 & .012 \\
\hline Estudios & .021 & .127 & .007 & .169 & .866 \\
\hline Trabajo & .285 & .409 & .026 & .697 & .486 \\
\hline Neuroticismo & -.149 & .031 & -.264 & -4.848 & .000 \\
\hline Extraversión & .082 & .030 & .117 & 2.734 & .006 \\
\hline Apertura & -.038 & .028 & -.054 & -1.349 & .178 \\
\hline Amabilidad & -.009 & .036 & -.010 & -.255 & .799 \\
\hline Responsabilidad & .097 & .030 & .133 & 3.269 & .001 \\
\hline Inteligencia Emocional & .030 & .007 & .238 & 4.067 & .000 \\
\hline (Constante) & 15.031 & 2.613 & & 5.752 & .000 \\
\hline Sexo & -.718 & .535 & -.049 & -1.341 & .180 \\
\hline Edad & -.042 & .022 & -.080 & -1.938 & .053 \\
\hline Estudios & -.003 & .121 & -.001 & -.028 & .978 \\
\hline Trabajo & .082 & .390 & .007 & .211 & .833 \\
\hline Neuroticismo & -.116 & .032 & -.205 & -3.618 & .000 \\
\hline Extraversión & .058 & .032 & .082 & 1.791 & .074 \\
\hline Apertura & -.033 & .027 & -.047 & -1.206 & .228 \\
\hline Amabilidad & .037 & .037 & .041 & 1.003 & .317 \\
\hline
\end{tabular}




\begin{tabular}{|c|c|c|c|c|c|}
\hline \multirow[t]{2}{*}{ Modelo } & \multicolumn{2}{|c|}{$\begin{array}{l}\text { Coeficientes no } \\
\text { estandarizados }\end{array}$} & \multicolumn{2}{|c|}{$\begin{array}{c}\text { Coeficientes } \\
\text { estandarizados }\end{array}$} & \multirow[b]{2}{*}{ Sig. } \\
\hline & $\mathrm{B}$ & Error Std. & Beta & $\mathrm{t}$ & \\
\hline Responsabilidad & .095 & .029 & .130 & 3.275 & .001 \\
\hline Conciencia emocional & .020 & .020 & .041 & .963 & .336 \\
\hline Regulación emocional & -.024 & .020 & -.057 & -1.162 & .246 \\
\hline Autonomía emocional & -.045 & .023 & -.092 & -1.922 & .000 \\
\hline Comp. Social & .010 & .028 & .018 & .364 & .716 \\
\hline Comp. de Vida & .182 & .022 & .423 & 8.279 & .055 \\
\hline
\end{tabular}

Nota. Var. Dependiente: Satisfacción con la vida. $\mathrm{N}=3073 \mathrm{R}^{2}$ para los pasos 1 a $4=.005$, .31, .33, y .39. Fuente: Elaboración propia.

Las ecuaciones de regresión: $\mathrm{SV}=7,907-0,108 \mathrm{e}-0,264 \mathrm{~N}+2,734 \mathrm{E}+$ $3,269 \mathrm{R}+4.067 \mathrm{IE} \mathrm{SV}=5,752-0,205 \mathrm{~N}+3,275 \mathrm{R}-0,092 \mathrm{AU}$

SV = Satisfacción en la vida, e = edad, N = Neuroticismo, E = Extroversión, $\mathrm{IE}=$ Inteligencia emocional, $\mathrm{AU}=$ Autonomía emocional.

\section{Discusión y conclusiones}

Este estudio evidencia la fiabilidad y validez del cuestionario CDE-A35 para medir la inteligencia emocional rasgo en adultos. Se presentan resultados que muestran la validez del constructo y también se confirma la validez incremental vis-à-vis con los cinco grandes rasgos de personalidad.

En cuanto a la validez del constructo, la fiabilidad interna de los cinco factores fue excelente. Se obtuvo una estructura con cinco escalas que coinciden con las dimensiones teóricas de conciencia emocional, regulación emocional, autonomía emocional, competencia social y competencias de vida del modelo pentagonal del GROP. Todas las escalas mostraron una alta fiabilidad y fuertes correlaciones con la medida global de IE.

Adicionalmente, la validez convergente del cuestionario se evidenció al encontrar fuertes asociaciones entre el CDE-A35 y el TElque. Las correlaciones son moderadas y elevadas, lo que es congruente con el hecho de que ambas medidas evalúen la Inteligencia emocional rasgo. Estos resultados apoyan la hipótesis de que el CDE-A35 mide lo que dice medir, es decir la inteligencia emocional rasgo de las personas. Paralelamente, la validez discriminante del cuestionario se evidenció por las elevadas 
correlaciones positivas con la escala de habilidades sociales EHS y con algunos componentes del cuestionario de pensamiento constructivo: autoaceptación, ausencia de sobregeneralización negativa, ausencia de hipersensibilidad, y ausencia de rumiaciones. Esta validez quedo reforzada por las correlaciones negativas con las escalas de pensamiento mágico, pensamiento categórico, y el pensamiento esotérico. Las correlaciones con el EHS ponen de manifiesto que la gestión efectiva de las emociones favorece el establecimiento y desarrollo de relaciones positivas y beneficiosas con los demás (Navarro, Varas y Maluenda, 2017).

Asimismo, la validez del constructo se vió reforzada por las correlaciones del CDE-A35 con las escalas de la personalidad. Confirmando las expectativas generadas por otros estudios (Fumero y Navarro, 2016), todas las escalas correlacionaron fuerte y negativamente con el neuroticismo y positivamente con la extroversión, la apertura, la amabilidad. Por su parte la competencia social y las competencias de vida, correlacionan fuertemente con la extraversión, de forma congruente con resultados de estudios anteriores (Moreira y Gamboa, 2015; Shafer, 1999). Las competencias de vida además correlacionan fuertemente con la responsabilidad y más moderadamente con la apertura. Parece pues que la percepción de llevar la vida bien enfocada y saber afrontar los retos no sólo requiere una estabilidad emocional, sino también una buena dosis de responsabilidad, amabilidad, y extraversión. Por otra parte, la conciencia emocional muestra correlaciones más fuertes con la responsabilidad y la apertura. Esto tiene sentido si se considera que el conocimiento de nuestras emociones y las emociones de los demás no necesariamente nos hace más estables emocionalmente, pero sí facilita un mejor entendimiento de los demás y permite estar más abierto a otras formas de ser y de hacer. Asimismo, un mayor conocimiento de nuestras propias emociones facilita un mayor compromiso con nuestros valores más profundos facilitando una mayor responsabilidad.

La validez de criterio se confirmó al comprobarse que el CDE-A35 predice una parte substancial de la varianza en la satisfacción con la vida. Individualmente, la regulación emocional, la autonomía emocional y las competencias de vida predicen la satisfacción en la vida.

Finalmente, como se intuía la IE medida por el CDE-A35 demostró su validez incremental prediciendo varianza en la satisfacción con la vida más allá de la que predicen las dimensiones de la personalidad. Aunque anteriores estudios (Lyons, Huebner y Hills, 2015) habían documentado 
asociaciones entre rasgos de personalidad y satisfacción con la vida, un componente de la IE produjo una mejora de la predicción de ésta. Por ello, los resultados sugieren que las competencias de vida permiten al individuo experimentar mayor satisfacción con la vida. Aunque la evidencia obtenida está restringida a una de las cinco escalas del CDE-A35 (con una segunda -autonomía emocional- aproximándose a la significación estadística), los resultados apoyan la hipótesis de que el CDE-A35 tiene capacidad de predicción de variables socialmente relevantes y es por tanto una herramienta de evaluación útil.

Conviene recordar que el estudio no está exento de limitaciones, así la muestra de este estudio está integrada mayoritariamente por mujeres, y posee un alto nivel cultural. En consecuencia, sería conveniente replicar estos resultados con nuevos estudios con muestras más representativas.

El CDE-A35 posee una buena fundamentación teórica basada en el modelo pentagonal del GROP (Bisquerra y Pérez, 2007) y la evidencia empírica aportada por este estudio nos permite afirmar que es un instrumento fiable, válido, útil y novedoso para medir la inteligencia emocional. Destacamos las aplicaciones educativas del CDE-A35 tanto en la evaluación de necesidades de desarrollo emocional como en el diseño y evaluación de intervenciones destinadas a la mejora de la IER. En este sentido, compartimos con Keefer (2015) que es de gran importancia disponer de un instrumento debidamente validado que permita conocer el nivel de desarrollo emocional y contribuir al diseño y evaluación de programas de intervención.

\section{Referencias}

Alegre, A., Pérez-Escoda, N. \& López-Cassà, E. (2019). The Relationship Between Trait Emotional Intelligence and Personality. Is Trait El Really Anchored Within the Big Five, Big Two and Big One Frameworks? Frontiers in Psychology, 10, 866. doi: 10.3389/ fpsyg.2019.00866

Andrei, F., Siegling, A. B., Aloe, A. M., Baldaro, B. \& Petrides, K.V. (2016). The incremental validity of the Trait Emotional Intelligence Questionnaire (TEIQue): a systematic review and meta-analysis. Journal of Personality Assessment, 98 (3), 261-276. doi: 10.1080/00223891.2015.1084630

Bisquerra, R. y Mateo, J. (2019). Competencias emocionales para un cambio de paradigma en educación. Barcelona: Horsori.

Bisquerra, R. y Pérez, N. (2007). Las competencias emocionales. Educación XX1, 10, 61 82. doi:10.5944/educxx1.10.1 
Pérez-Escoda, N., Alegre Rosselló, A. y López-Cassà, È. (2021). Validación y fiabilidad del cuestionario de desarrollo emocional en Adultos (CDE-A35). Educatio Siglo XXI, 39(3), 37-60.

Bisquerra, R., Pérez-González, J. C. y García Navarro, E. (2015). Inteligencia emocional en la educación. Madrid: Síntesis.

Cordero, A. (2001). CTI. Inventario de pensamiento constructivo. Una medida de la Inteligencia Emocional. Madrid: TEA. (Versión original en inglés: Epstein, S. Manual for the Constructive Thinking Inventory. Unpublished manuscript, University of Amherst, 1987).

Cordero, A., Pamos, A. y Seisdedos, N. (2008). NEO PI-R Manual. Adaptación Española ( $3^{a}$ edición). Madrid: TEA Ediciones.

Costa, P. y McCrae, R. (1989). Inventario de Evaluación de la Personalidad normal "NEO $P I-R$ " abreviado; "NEO-FFI". Madrid: TEA.

Cheing, G., Vong, S., Chan, F., Ditchman, N., Brooks, J. \& Chan, C. (2014). Testing a PathAnalytic Mediation Model of How Motivational Enhancement Physiotherapy Improves Physical Functioning in Pain Patients. Journal of Occupational Rehabilitation 24, 798-805. doi: 10.1007/s10926-014-9515-8

Diener, E., Emmons, R. A., Larsen, R. J. \& Griffin, S. (1985). The satisfaction with life scale. Journal of Personality Assessment, 49, 71-75. doi:10.1207/s15327752jpa4901_13

Diener, E. \& González, E. (2011). The validity of life satisfaction measures. Social Indicator Network News, 108, 1-5.

Durlak, J. A., Domitrovich, C. E., Weissberg, R. P. \& Gullotta, T. P. (2015). Handbook of Social and Emotional Learning. Research and Practice. Nueva York: The Guilford Press.

Fernández-Abascal, E. G. \& Martín-Díaz, M. D. (2015). Dimensions of emotional inteIligence related to physical and mental health and to health behaviors. Frontiers in Psychology, 6, 317. doi: 10.3389/fpsyg.2015.00317

Floyd, F. J. \& Widaman, K. F. (1995). Factor analysis in the development and refinement of clinical assessment instruments. Psychological Assessment, 7(3), 286-299. doi:10.1037/1040-3590.7.3.286

Fumero, A. y Navarro, G. (2016). Personalidad y Malestar Psicológico: Aplicación de un Modelo de Redes Neuronales. Revista Iberoamericana de Diagnóstico y Evaluación e Avaliação Psicológica. RIDEP, 41(1), 28-38.

Gismero, E. (2000). EHS. Escala de Habilidades Sociales. TEA Ediciones.

Hu, L. \& Bentler, P. M. (1995). Evaluating model fit. En R.H. Hoyle (Ed.). Structural equation modeling: Concepts, issues, and applications (pp.77-99). Thousand Oaks: Sage.

Hu, L.T. \& Bentler, P.M. (1999). Cutoff Criteria for Fit Indexes in Covariance Structure Analysis: Conventional Criteria Versus New Alternatives. Structural Equation Modeling, 6, 1-55. doi:10.1080/10705519909540118

Khayyam ,Z., Yousefy, A., Neshat, H., Manshaee, G. \& Sadeghei, M. (2014). Structural Model of psychological risk and protective factors affecting on quality of life in patients with coronary heart disease: A psychocardiology model. Journal of Research in Medical Sciences, 19, 90-98

Keefer, K. V. (2015). Self-report assessment of emotional competencies: A critical look at methods and meanings. Journal of Psychoeducational Assessment, 33, 3-23. doi:10.1177/0734282914550381

Keefer, K. V., Parker, J. D. A. \& Saklofske, D. H. (2018). Emotional Intelligence in Education. Integrating Research with Practice. Cham (Suiza): Springer. 
Pérez-Escoda, N., Alegre Rosselló, A. y López-Cassà, È. (2021). Validación y fiabilidad del cuestionario de desarrollo emocional en Adultos (CDE-A35). Educatio Siglo XXI, 39(3), 37-60.

Kline, R. B. (1998). Principles and practice of structural equation modeling. New York: Guilford Press.

Kotsou, I. (2019). Intelligence émotionnelle et management: comprendre et utiliser la force des émotions. Bruxelles: De Boeck.

Little, T. D. (2013). Longitudinal structural equation modeling. New York: Guilford Press.

Little, T. D., Rhemtulla, M., Gibson, K. \& Schoemann, A. M. (2013). Why the items versus parcels controversy needn't be one. Psychological Methods, 18 (3), 285 - 300. doi: $10.1037 / \mathrm{a} 0033266$

Lyons, M. D., Huebner, E. S. \& Hills, K. J. (2015). Relations among personality characteristics, environmental events, coping behavior and adolescents' life satisfaction. Journal of Happiness Studies, 17 (3), 1033-1050. doi:10.1007/s10902-015-9630-z

Magalhães, E., Salgueira, A., Gonzalez, A-J., Costa, J. J., Costa, M. J., Costa, P. \& Lima, M. P. (2014). NEO-FFI: Psychometric Properties of a Short Personality Inventory in Portuguese Context. Psychology/Psicologia Reflexão e Crítica, 27 (4), 642-657. doi: 10.1590/1678-7153.201427405

McCallum, R. C., Widaman, K. F., Zhang, S. \& Hong, S. (1999). Sample size in factor analysis. Psychological Methods, 4, 84-99. doi:10.1037/1082-989X.4.1.84

Moreira, J. M. y Gamboa, P. (2016). Inventário de Estados Afetivos-Reduzido: Uma Medida Multidimensional Breve de Indicadores Emocionais de Ajustamento. Revista Iberoamericana de Diagnóstico y Evaluación - e Avaliação Psicológica. RIDEP, 41(1), 132-144.

Navarro, G., Varas, M. y Maluenda J. (2017). Propiedades Psicométricas del Inventario de Asertividad de Gambrill y Richey en Estudiantes Universitarios Chilenos Revista Iberoamericana de Diagnóstico y Evaluación - e Avaliação Psicológica. RIDEP, 43 (1), 33-43. doi:10.21865/RIDEP43_33

O'Connor P.J., Hill A., Kaya M. \& Martin B. (2019). The Measurement of Emotional Intelligence: A Critical Review of the Literature and Recommendations for Researchers and Practitioners. Frontiers in Psychology, 10, 1116. doi: 10.3389/fpsyg.2019.01116

Osher, D., Kidron, Y., Brackett, M., Dymnicki, A., Jones, S. \& Weissberg, R. P. (2016). Advancing the Science and Practice of Social and Emotional Learning: Looking Back and Moving Forward. Review of Research in Education, 40 (1), 644-681. doi:10.3102/0091732X16673595

Pérez-Escoda, N. y Filella, G. (2019). Educación emocional para el desarrollo de competencias emocionales en niños y adolescentes. Praxis \& Saber, 10 (24), 23-44. doi: 10.19053/22160159.v10.n25.2019.8941

Pérez-González, J. C. \& Sánchez-Ruiz, M. J. (2014). Trait emotional intelligence anchored within the Big Five, Big Two and Big One frameworks. Personality and Individual Differences, 65, 53-58. doi:10.1016/j.paid.2014.01.021

Petrides, K. V., Mikolajczak, M., Mavroveli, S., Sanchez-Ruiz, M. J., Furnham, A. \& PérezGonzález, J. C. (2016). Developments in trait emotional intelligence research. Emotion Review, 8 (4), 335-341. doi:10.1177/1754073916650493

Petrides K.V., Sanchez-Ruiz MJ., Siegling A.B., Saklofske D.H. \& Mavroveli S. (2018) Emotional Intelligence as Personality: Measurement and Role of Trait Emotional Intelligence in Educational Contexts. En K. Keefer, J.Parker y D. Saklofske (Eds.). Emo- 
tional Intelligence in Education. Cham:The Springer Series on Human Exceptionality, Springer.

Quintana, S. M. \& Maxwell, S. E. (1999). Implications of recent developments in structural equation modeling for counseling psychology. The Counseling Psychologist, 27 (4), 485-527. doi:10.1177/0011000099274002

Sánchez-Ruiz, M. J., Pérez-González J.C. \& Petrides K. V. (2010). Trait emotional inteIligence profiles of students from different university faculties, Australian Journal of Psychology, 62 (1), 51-57. doi:10.1080/00049530903312907

Sánchez-Teruel, D. y Robles-Bello, M. A. (2018). Instrumentos de evaluación en inteligencia emocional: una revisión sistemática cuantitativa. Perspectiva Educacional, 57(2), 27-50. doi: 10.4151/07189729-vol.57-iss.2-art.712

Shafer, A. B. (1999). Relation of the Big Five and Factor $V$ subcomponents to social inteIligence. European Journal of Personality, 13 (3), 225-240. doi:10.1002/(SICl)10990984(199905/06)13:3<225::AID-PER337>3.0.CO;2-V

Vázquez, C., Duque, A. \& Hervas, G. (2013). Satisfaction with life scale in a representative sample of Spanish adults: Validation and normative data. The Spanish Journal of Psychology, 16, E82-E94. doi:10.1017/sjp.2013.82 